\begin{tabular}{|c|l|}
\hline Title & Dynamical conductivity in the multiply degenerate point-nodal semimetal CoSi \\
\hline Author(s) & Habe, Tetsuro \\
\hline Citation & $\begin{array}{l}\text { Physical Review B, 100(24), 245131 } \\
\text { https://doi.org/10.1103/PhysRevB.100.245131 }\end{array}$ \\
\hline Issue Date & 2019-12-18 \\
\hline Doc URL & http://hdl.handle.net/2115/76639 \\
\hline Rights & Copyright (2019) by The A merican Physical Society. \\
\hline Type & article \\
\hline File Information & PhysRevB.100.245131.pdf \\
\hline
\end{tabular}

Instructions for use 


\title{
Dynamical conductivity in the multiply degenerate point-nodal semimetal CoSi
}

\author{
Tetsuro Habe (1) \\ Department of Applied Physics, Hokkaido University, Sapporo, Hokkaido 060-0808, Japan
}

(Received 18 March 2019; published 18 December 2019)

\begin{abstract}
We theoretically investigate the dynamical conductivity in the multiply degenerate point-nodal semimetal $\mathrm{CoSi}$. The dynamical conductivity is calculated as a function of photon energy by using the first-principles band calculation and the linear response theory. In the nodal semimetal, the band structure holds point nodes at the $\Gamma$ and $\mathrm{R}$ points in the Brillouin zone and more than three bands touch at the nodes. Around the nodes, electronic states are predicted to be described as the multifold chiral fermion, a class of fermion in condensed matter. We show that the dynamical conductivity exhibits a characteristic spectrum corresponding to the band structure and the chiral fermionic states. The chirality leads to the prohibition of transition between the lower and upper bands of threefold chiral fermion and thus the transition between the middle and lower bands is relevant to the dynamical conductivity. This transition property is different from the Dirac and Weyl semimetals, the other point-nodal semimetals, where the excitation between the upper and lower bands is relevant to the dynamical conductivity. We show the prohibition causes the reduction of dynamical conductivity in the low-photon energy region.
\end{abstract}

DOI: 10.1103/PhysRevB.100.245131

\section{INTRODUCTION}

Topological nodal semimetals have attracted much attention in condensed-matter physics due to the electronic structure and the unique phenomena associated with them. In point-nodal semimetals, the conduction and valence bands touch each other at points, called nodes, in the Brillouin zone [1]. Dirac and Weyl semimetals are the first generation of topological point-nodal semimetals [2-6]. In these semimetals, electronic states around the nodes are described by Dirac equation in high-energy physics and have provided a test ground for studying the property of Dirac and Weyl fermions in condensed matter. Recently, a type of point-nodal semimetals has been proposed theoretically and predicted to have multiply degenerate electronic states, so-called multifold chiral fermions, which cannot be described by Dirac equation [7-11]. The chiral fermion can emerge in some crystals as a robust electronic state topologically protected [12]. Such topological nodal semimetals have been expected to show novel electronic properties beyond Dirac fermions. For instance, the unique surface states [13-15] and the quantized photogalvanic response $[13,16,17]$ have been studied theoretically and experimentally.

$\mathrm{CoSi}$ is a strong candidate of novel topological semimetals according to the first-principles band calculation $[10,12,18,19]$. Angle-resolved photoemission spectroscopy (ARPES) is one of the experimental techniques for identifying the band structure of topological semimetals [5,6]. Recently, a few groups have reported the band structure of $\mathrm{CoSi}$ by using ARPES and shown nodes and topological surface states, one of the characteristic features of the topological semimetal $[20,21]$. However, there is a restriction in the visible electronic energy for ARPES because it is able to detect electronic states up to the Fermi level. In the pristine crystal of the semimetal, the Fermi level is pinned at the charge neutral point, and it is slightly under one of the nodes in $\mathrm{CoSi}$.
In this paper, we theoretically investigate the dynamical conductivity of $\mathrm{CoSi}$ by using the first-principles calculation and the linear response theory. The dynamical conductivity is associated with interband transitions between the occupied and unoccupied bands, and thus it provides information about electronic bands above the Fermi level. The photon energy dependence of dynamical conductivity is strongly affected by the band structure. In Ref. [22], Habe and Kohino showed that the numerical result by using the first-principles band calculation agrees well with the experimental measurement of dynamical conductivity for a topological nodal-line semimetal [23]. By using the method, in this paper, we provide the realistic numerical result comparable to experimental data and reveal the relation between the spectrum of dynamical conductivity and the electronic states in CoSi. Especially below $250 \mathrm{meV}$, we find that the spectrum is ascribable to not only the band structure but also the property of multifold chiral fermions; a prohibition of electronic transition between a specific pair of bands. The multifold chiral fermion imposes the reduction of dynamical conductivity and causes a dip structure in the spectrum around the photon energy of $200 \mathrm{meV}$.

\section{FIRST-PRINCIPLES BAND STRUCTURE}

We calculate the electronic band structure of CoSi by using QUANTUM ESPRESSO, a first-principles calculation code [24], and show it in Fig. 1(c). The cubic unit cell and the Brillouin zone are schematically depicted in Figs. 1(a) and 1(b), respectively. The lattice constant and the atomic position are computed within the same code by relaxing the lattice to the stable structure. Then, the lattice constant is estimated to be $4.432 \AA$ and the atomic position is given in Table I. The crystal structure is classified into $P 2_{1} 3$, the symmetry group (SG) 198, and represented by two parameters of $x_{\mathrm{Co}}=0.144$ 
(a)
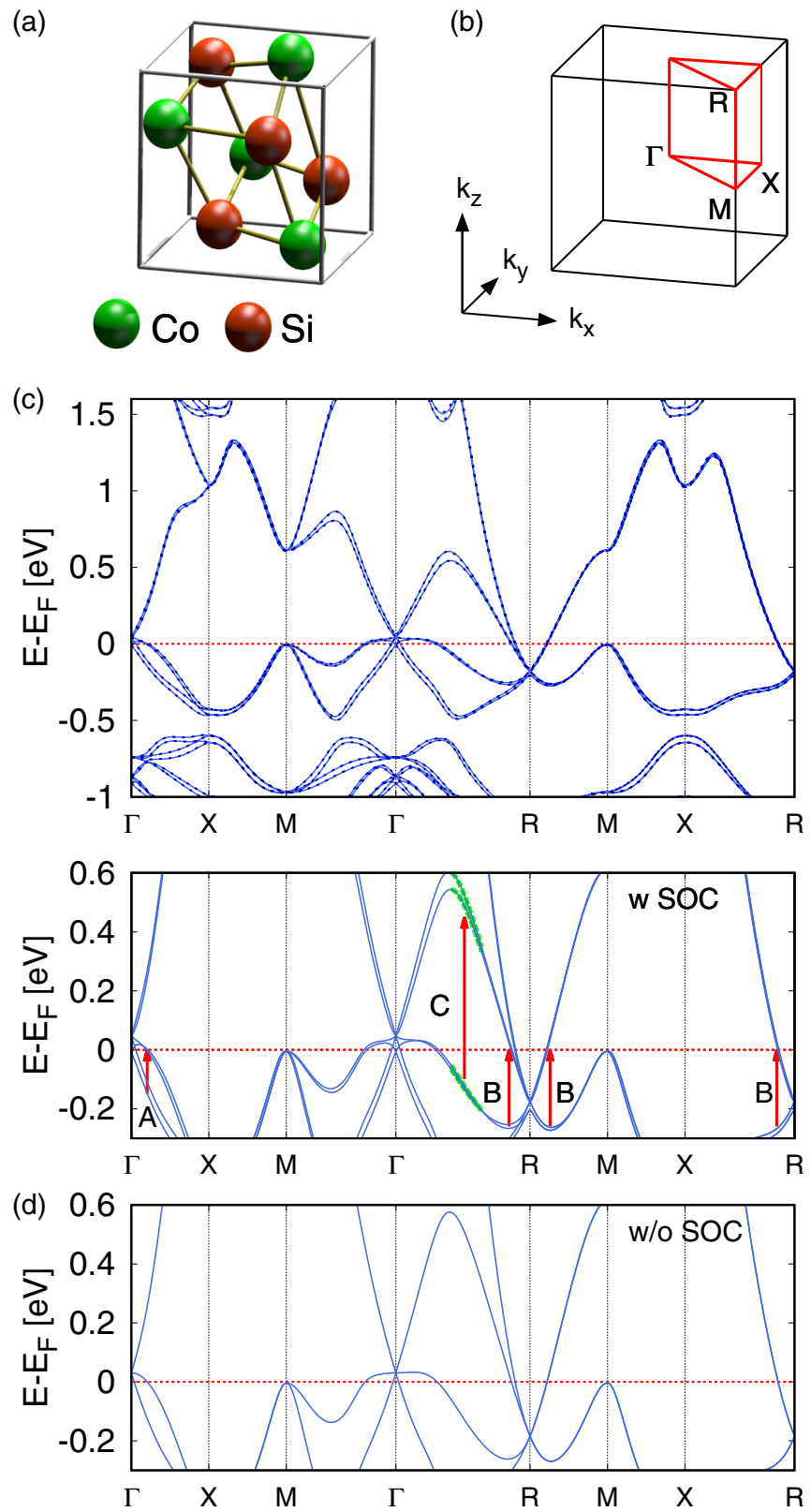

FIG. 1. The unit cell and the Brillouin zone for CoSi in (a) and (b), respectively. The band structure of CoSi calculated by the firstprinciples calculation, including the spin-orbit coupling in (c). The band structure within different energy ranges is depicted in the upper and lower panels. The dashed line in the upper panel indicates the band calculated by using the tight-binding model discussed in Sec. III. In the lower panel, arrows A, B, and C indicate the transition processes corresponding to the characteristic structure of dynamical conductivity in Fig. 3(a). The highlighted region is associated with the first peak around $650 \mathrm{meV}$ in Fig. 2. In (d), the band is calculated without the spin-orbit interaction.

and $x_{\mathrm{Si}}=0.843$ for Co and $\mathrm{Si}$, respectively. We apply a projector augmented-wave method to the first-principles calculation with a generalized-gradient approximation functional, including spin-orbit coupling (SOC). The cutoff energy of the plane-wave basis and the convergence criterion are adopted as 50 Ry and $10^{-8}$ Ry, respectively.
TABLE I. The list of atomic positions in the unit cell of CoSi, which is shown in Fig. 1(a). The positions are represented in units of the lattice constant.

\begin{tabular}{lccc}
\hline \hline & $x$ & $y$ & $z$ \\
\hline $\mathrm{Co}^{(1)}$ & 0.144 & 0.144 & 0.144 \\
$\mathrm{Co}^{(2)}$ & 0.644 & 0.356 & 0.856 \\
$\mathrm{Co}^{(3)}$ & 0.356 & 0.856 & 0.644 \\
$\mathrm{Co}^{(4)}$ & 0.856 & 0.644 & 0.356 \\
$\mathrm{Si}^{(1)}$ & 0.843 & 0.843 & 0.843 \\
$\mathrm{Si}^{(2)}$ & 0.343 & 0.657 & 0.157 \\
$\mathrm{Si}^{(3)}$ & 0.657 & 0.157 & 0.343 \\
$\mathrm{Si}^{(4)}$ & 0.157 & 0.343 & 0.657 \\
\hline \hline
\end{tabular}

We show the first-principles band structure of $\mathrm{CoSi}$ in Fig. 1(c), where is presented within the different energy ranges in the upper and lower panels. Nodal points appear at two high-symmetry points: The $\Gamma$ point and the R point. At the $\Gamma$ point, electronic states are quadruply degenerated at the upper node and doubly degenerated at the lower node, where the slight energy split between nodes is due to SOC [25]. In the absence of SOC, a single node appears with triply degenerated electronic states for each spin as shown in Fig. 1(d). The fourfold degenerated node is slightly above the Fermi level, and a doubly degenerate node emerges under the level. At the R point, a single node appears with sextuple degeneration $175 \mathrm{meV}$ below the Fermi energy. Four cones and a single quadratic band are crossing at the node. In the band structure without SOC, a fourfold degenerate node emerges for each spin at the $\mathrm{R}$ point.

\section{DYNAMICAL CONDUCTIVITY}

The dynamical conductivity $\sigma(\omega)$ is the response function to the oscillating electric field with the frequency $\omega$ and is related to the optical property of materials. The real part $\sigma_{1}(\omega)=\operatorname{Re}[\sigma(\omega)]$ is proportional to the optical absorption. In experiments, $\sigma_{1}(\omega)$ is obtained from the reflectivity of a single crystal with the polish surface under the normal incident photon by Kramers-Kronig analysis. The photon-energy dependence of $\sigma_{1}$ shows the unique spectrum depending on the band structure of nodal semimetal; a flat spectrum for graphene [26-28], a linear dependence for Dirac and Weyl semimetals [29-31], and a nearly flat spectrum for nodal-line semimetals $[22,32,33]$. These spectra have been observed in some experimental fabrications $[23,34,35]$.

In general, $\sigma_{1}(\omega)$ is associated with the electronic transition corresponding to the frequency of applied electric field, i.e., the photon energy. We represent the electronic states in $\mathrm{CoSi}$ by using a multiorbital tight-binding model defined on the basis of Wannier orbitals. We adopt five $d$ orbitals in Co and three $p$ orbitals in $\mathrm{Si}$ as the basis and compute the spin-dependent hopping integrals and on-site potential from the first-principles bands in Fig. 1 by using WANNIER90, a code for calculating maximally localized Wannier functions and the hopping matrix [36]. This tight-binding model reproduces the first-principles band structure as shown in Fig. 1. We calculate the dynamical conductivity in the linear response theory where, for a linearly polarizing photon, it is 

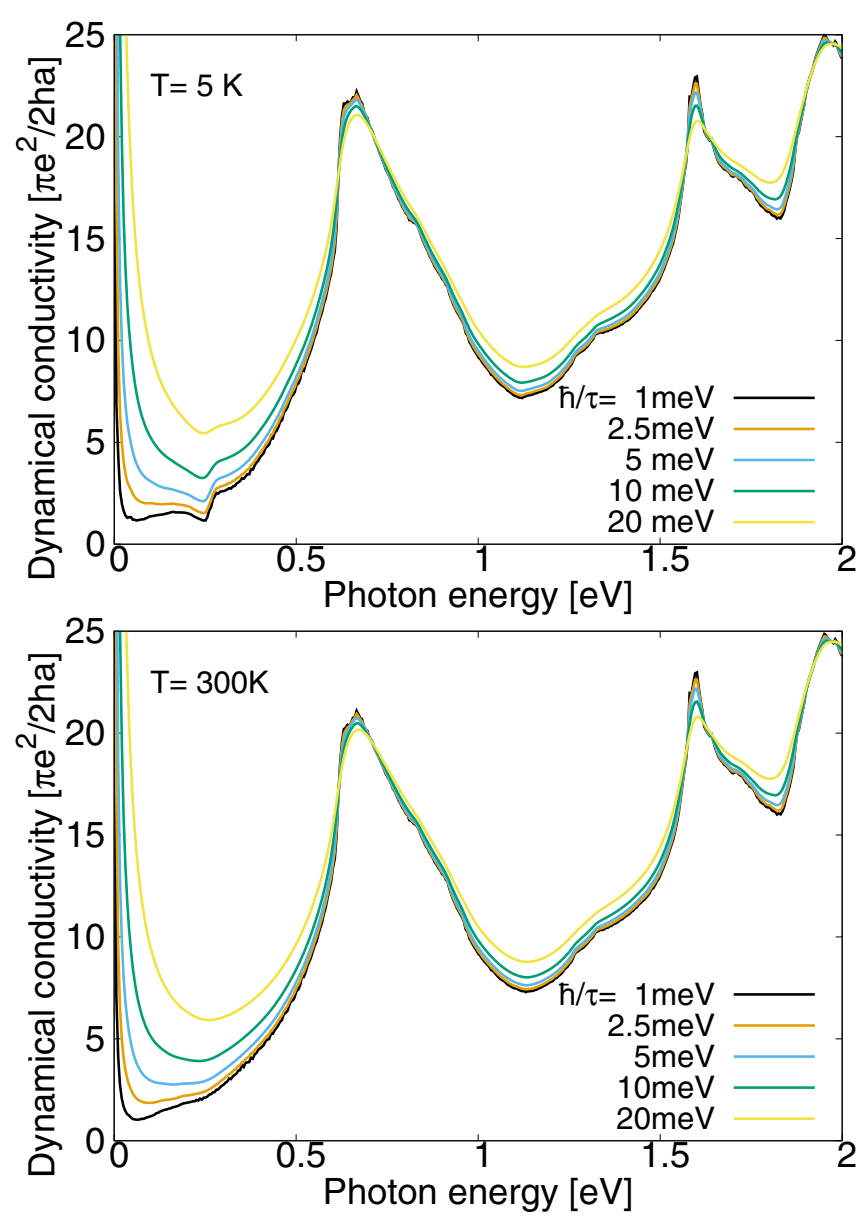

FIG. 2. The dynamical conductivity as a function of photon energy plotted for several relaxation times, $\hbar / \tau=1,2.5,5,10$, and $20 \mathrm{meV}$, at $5 \mathrm{~K}$ in the upper panel and at $300 \mathrm{~K}$ in the lower panel.

represented by

$$
\begin{aligned}
\sigma(\omega, T)= & \frac{2 i}{\omega} \frac{e^{2}}{\hbar} \sum_{m \leqslant n} \int_{\mathrm{BZ}} \frac{d^{3} \boldsymbol{k}}{(2 \pi)^{3}} \frac{\left|\left\langle n \boldsymbol{k}\left|\hat{v}_{\alpha}\right| m \boldsymbol{k}\right\rangle\right|^{2}}{\hbar \omega-\left(E_{n \boldsymbol{k}}-E_{m \boldsymbol{k}}\right)+i \hbar /(2 \tau)} \\
& \times\left(n_{F}\left(E_{n \boldsymbol{k}}, T\right)-n_{F}\left(E_{m \boldsymbol{k}}, T\right)\right)
\end{aligned}
$$

where $n_{F}(E, T)$ is Fermi distribution function at temperature $T$ and energy $E$ with respect to the Fermi level. Here, $\omega$ is the frequency of photon and the velocity is defined by the commutation of the position and the Hamiltonian, $\hat{v}_{\alpha}=$ $(1 / i \hbar)\left[x_{\alpha}, H\right]$, where $\alpha$ indicates a parallel direction to the polarizing direction of photon. The eigenstate $|m \boldsymbol{k}\rangle$ and the energy $E_{m k}$ are calculated by using the multiorbital tightbinding Hamiltonian. The effect of impurity is included in Eq. (1) as the relaxation time $\tau$.

We consider the dynamical conductivity for linearly polarized photons in the (100) direction and show the relaxation time dependence of $\sigma_{1}(\omega)$ at $T=5 \mathrm{~K}$ and $300 \mathrm{~K}$ in Fig. 2. The low-frequency spectrum is sensitive to $\tau$ in $\omega<500 \mathrm{meV}$ because the tail of the Drude peak around $\omega=0$ spreads with the increase in the impurity density, i.e., the decrease of relaxation time $\tau$. When the material is clean, the interband transition is relevant to the spectrum in the low-frequency region. In the nearly ballistic condition, $\hbar / \tau=1 \mathrm{meV}$, the
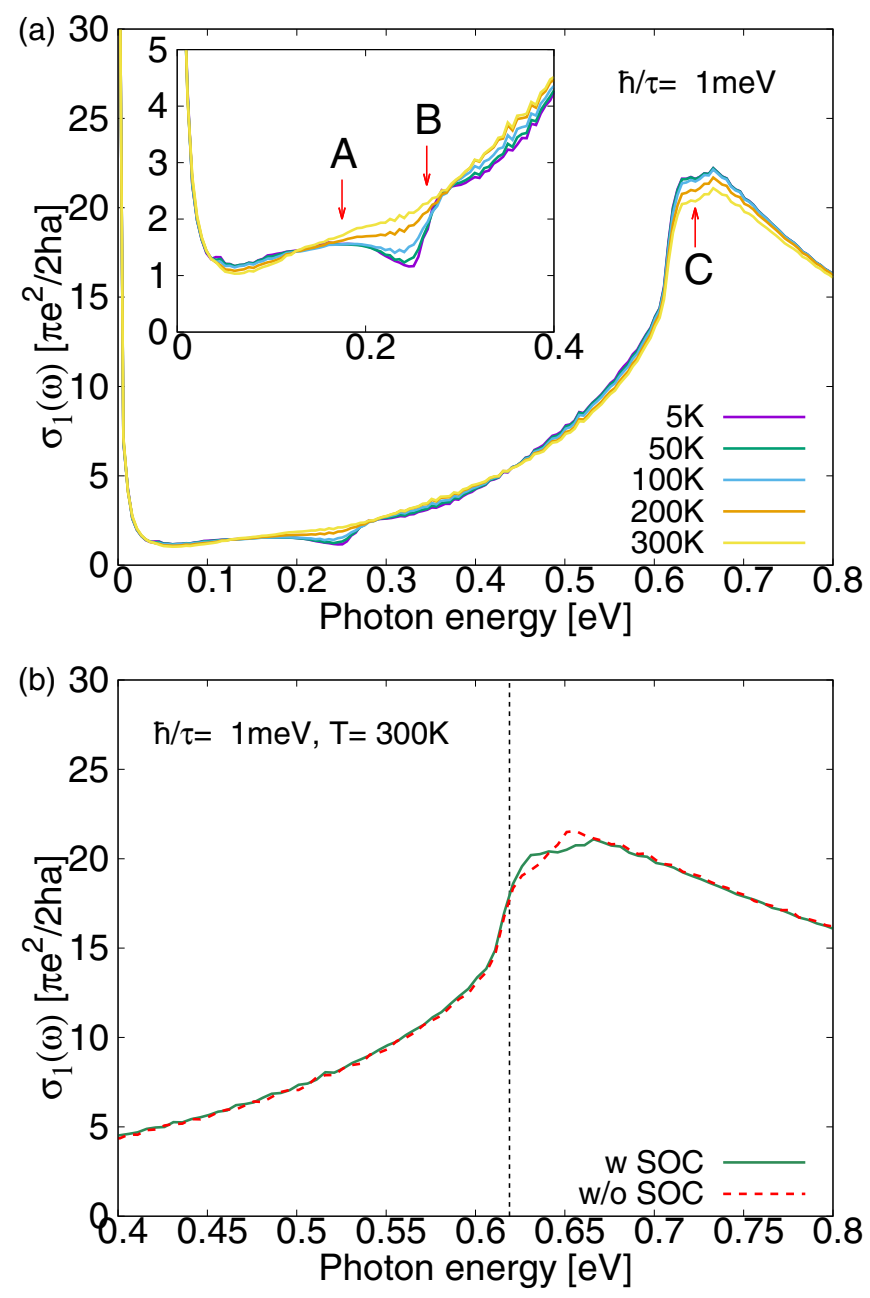

FIG. 3. The dynamical conductivity plotted at several temperatures, $T=5,10,50,100$, and $300 \mathrm{~K}$, for $\hbar / \tau=1 \mathrm{meV}$ and 10 $\mathrm{meV}$ in (a). A, B, and $\mathrm{C}$ correspond to the excitation energies in the transition processes indicated in Fig. 1(c). In (b), the dynamical conductivity calculated with and without the spin-orbit coupling. The vertical dashed line indicates the minimal excitation energy at the $\mathrm{M}$ point.

dynamical conductivity linearly increases with $\omega$ up to $\omega \sim$ $150 \mathrm{meV}$ at both the temperatures. The linear dependence is a characteristic feature of three-dimensional point-nodal semimetals and attributed to the density of states [14,29]. This characteristic structure disappears with the increase in $\hbar / \tau$, i.e., the increase of disorder, due to the broadening of the Drude peak, but it is stable in a clean sample even at the room temperature.

In Fig. 3, we show the temperature dependence of $\sigma_{1}(\omega)$ up to the room temperature with $\hbar / \tau=1 \mathrm{meV}$ in a narrower energy window. The numerical result exhibits that the linear slope is unchanged with the increase in temperature. In what follows, we investigate the structures of spectrum in this energy region and characterize them by the electronic transition in the band structure.

First, we find a dip structure emerging around $\hbar \omega \sim$ $250 \mathrm{meV}$ in the spectrum of $\sigma_{1}(\omega)$ below $200 \mathrm{~K}$. The steplike upward slope and the downward slop are attributed to 
different chiral fermions at the $\Gamma$ point and the R point in the Brillouin zone. This dip structure is different from a similar structure associated with a single chiral fermion in a recent work [14]. The dip structure in the previous work appears at the photon frequency corresponding to the energy of the node with respect to the Fermi level, $\hbar \omega \sim 10 \mathrm{meV}$. In the present paper, however, the dip structure appears at much larger photon energy, $\hbar \omega<100 \mathrm{meV}$. In what follows, we discuss the upward and downward slopes of the dip structures separately.

The upward slope emerges with a steplike structure at the photon energy B in Fig. 3(a). The photon energy is corresponding to the excitation energy to electronic states in the Fermi level around the $\mathrm{R}$ point, where the transition is depicted as B in Fig. 1. In general, the dynamical conductivity is a linear function of photon energy in the case of threedimensional linear dispersion with the Fermi level crossing the node [29]. When the upper (lower) cone is partially filled, there is no transition to electronic states, which are occupied, in the upper (lower) cone below (above) the Fermi level. Thus the dynamical conductivity is zero below the photon energy corresponding to the transition to the Fermi level and shows a steplike upward slope at the energy [37]. Therefore, the upward slope is attributed to the partially filled multifold cone at the $\mathrm{R}$ point.

The downward slope and the lower spectrum, on the other hand, are ascribable to the multifold chiral fermion at the $\Gamma$ point. Since the excitation energy is smaller than that of $\mathrm{B}$, the multifold cone at the $\mathrm{R}$ point has no contribution. To confirm the relation to the chiral fermions, we consider an effective Hamiltonian describing electronic states around the $\Gamma$ point and compare the dynamical conductivity in two models. Here we omit the SOC in the effective Hamiltonian because the low-energy dynamical conductivity is nearly unchanged with the presence of SOC. The effective Hamiltonian is represented by a $3 \times 3$ matrix on the basis of three degenerated electronic states $\Psi_{j}$ for $j=1-3$ at the $\Gamma$ point. The constant element gives the energy of the node at the $\Gamma$ point and thus it is proportional to the identity matrix. We consider only the matrix element linearly depending on the wave number $k_{\mu}$ in the effective model. Such a $k_{\mu}$-linear element can be represented by $\left\{H_{3 \times 3}\right\}_{i j}=\sum_{\mu} k_{\mu}\left\langle\Psi_{i}\left|V_{\mu}\right| \Psi_{j}\right\rangle$, where $V_{\mu}$ is defined by the derivative of the full Hamiltonian $\hbar^{-1} \partial H / \partial k_{\mu}$ and $\left|\Psi_{j}\right\rangle$ is the wave function at the $\Gamma$ point. Here $V_{\mu}$ and $\left|\Psi_{j}\right\rangle$ are calculated numerically by using the Fourier transformed multiorbital tight-binding Hamiltonian.

We show the band structure and the dynamical conductivity calculated by using the effective Hamiltonian in Figs. 4(a) and 4(b), respectively. The wave vector is confined in a cubic region $k_{\alpha} \in[-2 \pi /(10 a), 2 \pi /(10 a)]$ for $\alpha=x, y$, and $z$ where the middle band can be approximated to be a flatband and unoccupied. The dynamical conductivity is also calculated by using the effective Hamiltonian and shown at $\hbar / \tau=1 \mathrm{meV}$ and $T=50 \mathrm{~K}$ in Fig. 4(b). The structure of the spectrum qualitatively agrees with that in Fig. 3(a). The spectrum has a peak at $\omega=170 \mathrm{meV}\left(\mathrm{A}^{\prime}\right)$ and vanishes around $300 \mathrm{meV}($ ). In Fig. 4, we indicate two transition processes corresponding to $\mathrm{A}^{\prime}$ and . Both transitions occur at the edge of the cubic region and the excitation energy is minimum (maximum) at $\mathrm{A}^{\prime}()$ in the edge.

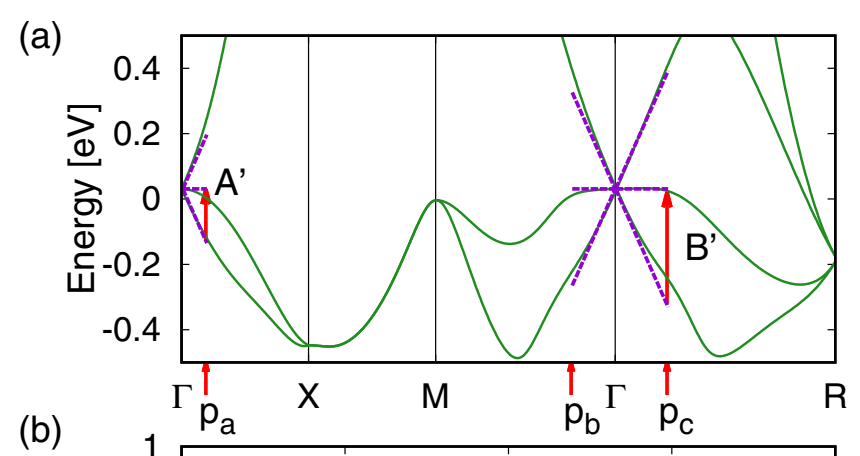

(b)

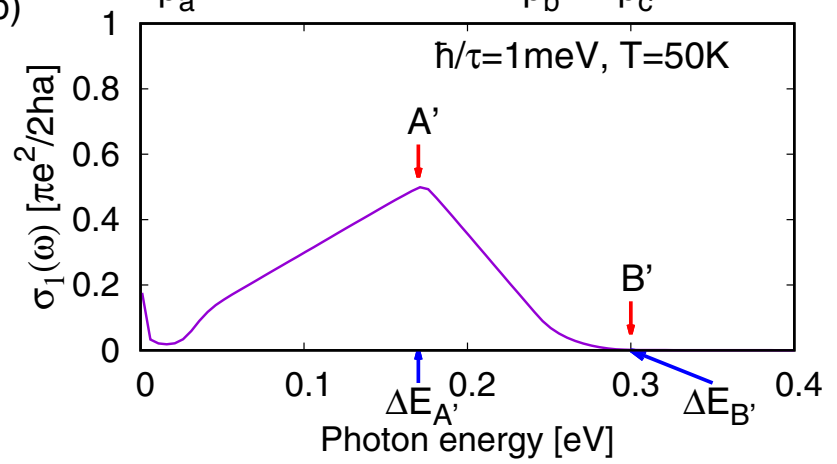

FIG. 4. The band structure (a) and the dynamical conductivity (b) calculated by using the effective model. The dashed line indicates the band structure calculated by using $k$-linear effective Hamiltonian. The Drude peak is eliminated in the calculation. Here, $\Delta E_{\alpha}$ is the excitation energy in the transition $\alpha=\mathrm{A}^{\prime}$ and $\mathrm{B}^{\prime}$.

The spectrum of dynamical conductivity is associated with the number of states contributing to the electronic transition. In Fig. 5, we schematically depict the wave vector at which the electronic transition between the middle and lower bands occurs for the photon energy $\hbar \omega$. The square region corresponds to the slice of cubic region for the effective Hamiltonian. Since the energy dispersion of the effective Hamiltonian is isotropic, the corresponding wave vectors appear on a spherical shell with the radius $k=\hbar \omega / v$, where $v$ is the gradient of lower band. The area of spherical shell, the number of electronic states contributing to the dynamical conductivity, increases with $\omega$ as far as $\hbar \omega \leqslant \Delta E_{\mathrm{A}^{\prime}}$, where $\Delta E_{\mathrm{A}^{\prime}}$ is the excitation energy in the transition $\mathrm{A}^{\prime}$. For $\Delta E_{\mathrm{A}^{\prime}}<\hbar \omega$, on the other hand, the area decreases with $\omega$ because the diameter of sphere

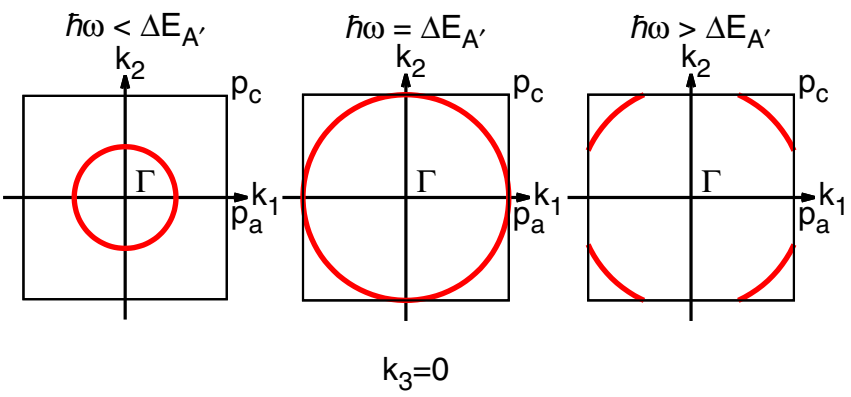

FIG. 5. The distribution of electronic states corresponding to the relevant transition for the dynamical conductivity in the effective model. Three panels schematically represent the case of different photon energy $\hbar \omega$. The wave numbers $\left(k_{1}, k_{2}, k_{3}\right)$ are any permutation of $\left(k_{x}, k_{y}, k_{z}\right)$. Here $p_{j}$ is corresponding to those in Fig. 4(b). 
exceeds the side of the cubic region. Since the square region simulates the wave vectors at which the middle band is unoccupied, the peak structure $\mathrm{A}^{\prime}$ is dominated by the electronic transition between the lower cone and the middle band.

The spectrum calculated by using the effective model implies the absence of transition between the upper and lower cones. The excitation energy between the two cones is larger than that between the lower and middle bands, and it is also within the high frequency region $\Delta E_{\mathrm{B}^{\prime}}<\hbar \omega$ but the dynamical conductivity vanishes for $\Delta E_{\mathrm{B}^{\prime}}<\hbar \omega$. Here, $\Delta E_{\mathrm{B}^{\prime}}$ is the maximum excitation energy between the lower and middle bands. Thus, this result means the prohibition of transition between the lower and upper bands. This prohibition is a unique feature of the chiral fermion and essentially different from the case of the Dirac fermion, where the intercone excitation is relevant to the dynamical conductivity [26]. In the next section, we confirm the prohibition for threefold chiral fermions analytically.

Second, we focus on a peak with a flat top around $\omega \simeq$ $650 \mathrm{meV}$. This flat-top peak is attributed to the parallel structure of bands and exhibits the effect of SOC to the nodal band structure around the $\mathrm{R}$ point. We represent the electronic states associated with the relevant transition to the dynamical conductivity as a shaded line in Fig. 1(c). The occupied and unoccupied states are distributed in the aligned lines of band structure. In the aligned occupied and unoccupied bands, electronic states show the resonant transition at nearly the same photon energy and produce a peak structure similar to the case of the nodal-line semimetal [22]. Moreover, the spin split of the upper band affects the structure of the peak. In practice, the peak structure changes with the presence of SOC, as shown in Fig. 3(b). In general, the spin split of the band leads to the split of the peak in the spectrum of dynamical conductivity because the spin split leads to two resonant states. However, the spin split in the parallel bands does not split the peak structure. Since the split gradually decreases to zero with the wave number toward the $\mathrm{R}$ point, electronic states have different excitation energies distributed in a range corresponding to the SOC split and they produce a flat-top peak consisting of a series of peaks with the energy continuously changing. Therefore, the flat-top peak exhibits the SOC split in the band of multifold chiral fermion at the $\mathrm{R}$ point.

Finally, in a higher-frequency region, we find the other peaks at $\omega \simeq 1.6 \mathrm{eV}$ and $1.9 \mathrm{eV}$. The two peaks are attributed to the enhancement of density of states at the local maximum and minimum of energy dispersion at high symmetry points. The peak at $1.6 \mathrm{eV}$ is corresponding to the electronic transition between the lowest conduction band and the second highest valence band around $-1 \mathrm{eV}$ at the $\mathrm{M}$ point in Fig. 1(c). Another peak emerges at $1.9 \mathrm{eV}$ due to the excitation between the highest valence band and the band slightly above $1.5 \mathrm{eV}$ at the $\mathrm{X}$ point. However, the electronic transition between the lowest conduction band and the highest valence band is absent at both the $\mathrm{X}$ and $\mathrm{M}$ points. This is because electronic states at these points have the same eigenvalue for twofold rotation operator $R_{1 / 2}$ along the $x, y$, and $z$ axes where twofold screw rotation is equivalent to twofold rotation at these highsymmetry momenta. The eigenvalue of the twofold rotation operator is \pm 1 because of $R_{1 / 2}{ }^{2}=1$. The states with the eigenvalue $1(-1)$ consist of Wannier orbitals with even (odd) angular momenta. Thus, electronic transition between the states with the same eigenvalue is prohibited because photons change the angular momentum by one.

\section{EFFECTIVE THEORY}

In this section, we analyze the electronic transition probability around the $\Gamma$ point in the low-frequency region by using an effective model based on the crystal symmetry of $\mathrm{CoSi}$. We ignore the effect of SOC in this analysis because the coupling does not have a relevant contribution to the dynamical conductivity in this energy region, according to the numerical calculation. In this analysis, we introduce a symmetry under the operation combining inversion and complex conjugation and show that the symmetry leads to the prohibition of electronic transition between the upper and lower bands of multifold chiral fermion around the $\Gamma$ point in $\mathrm{CoSi}$.

At the $\Gamma$ point, three electronic states are degenerate and the degenerated states are bases of an irreducible representation $T$ because CoSi is classified into $P 2_{1} 3$ (SG 198). The crystal structure has three axes of twofold screw symmetry(100), (010), and (001) — and four axes of threefold rotation

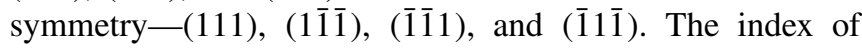
the representation is $\chi=3$ for identity operation, $\chi=-1$ for twofold screw rotation, and $\chi=0$ for threefold rotation. In what follows, we consider wave functions consisting of Wannier orbitals in the representation $T$.

The first-principles calculation reveals that the $d$ orbitals in Co atoms constitute a significant part of the electronic states around the Fermi level. In the unit cell, there are four Co atoms at $\boldsymbol{d}_{j}$, where the atomic positions are given by

$$
\begin{aligned}
& \boldsymbol{d}^{(1)}=\left(x_{\mathrm{Co}}+1 / 4, x_{\mathrm{Co}}-1 / 2, x_{\mathrm{Co}}\right), \\
& \boldsymbol{d}^{(2)}=\left(x_{\mathrm{Co}}-1 / 4,-x_{\mathrm{Co}},-x_{\mathrm{Co}}\right), \\
& \boldsymbol{d}^{(3)}=\left(-x_{\mathrm{Co}}-1 / 4,-x_{\mathrm{Co}}+1 / 2,-1 / 2+x_{\mathrm{Co}}\right), \\
& \boldsymbol{d}^{(1)}=\left(-x_{\mathrm{Co}}+1 / 4, x_{\mathrm{Co}}, 1 / 2-x_{\mathrm{Co}}\right) .
\end{aligned}
$$

Here we shift the origin of coordinate for the clear explanation about the symmetry in comparison with Table I. The wave function can be a superposition of Wannier orbitals at the four positions and it must fulfill the crystal symmetries of CoSi; twofold screw rotation and threefold rotation. Twofold screw rotation about (100) direction exchanges these atoms as $\left(\boldsymbol{d}^{(1)}, \boldsymbol{d}^{(2)}, \boldsymbol{d}^{(3)}, \boldsymbol{d}^{(4)}\right) \rightarrow$ $\left(\boldsymbol{d}^{(3)}, \boldsymbol{d}^{(4)}, \boldsymbol{d}^{(1)}, \boldsymbol{d}^{(2)}\right)$. Threefold rotation about (111) leads to $\left(d^{(1)}, d^{(2)}, d^{(3)}, d^{(4)}\right) \rightarrow\left(d^{(1)}, d^{(3)}, d^{(4)}, d^{(2)}\right)$ with the rotation angle $\theta=2 \pi / 3$. Then we can adopt three degenerated states at the $\Gamma$ point as

$$
\begin{aligned}
\Psi_{1}(\boldsymbol{r})= & \frac{1}{2}\left\{\psi_{m}^{(111)}\left(\boldsymbol{r}-\boldsymbol{d}^{(1)}\right)-\psi_{m}^{(1 \overline{1} \overline{1})}\left(\boldsymbol{r}-\boldsymbol{d}^{(2)}\right)\right. \\
& \left.+\psi_{m}^{(\overline{1} \overline{1} 1)}\left(\boldsymbol{r}-\boldsymbol{d}^{(3)}\right)-\psi_{m}^{(\overline{1} 1 \overline{1})}\left(\boldsymbol{r}-\boldsymbol{d}^{(4)}\right)\right\}, \\
\Psi_{2}(\boldsymbol{r})= & \frac{1}{2}\left\{\psi_{m}^{(111)}\left(\boldsymbol{r}-\boldsymbol{d}^{(1)}\right)+\psi_{m}^{(1 \overline{1} \overline{1})}\left(\boldsymbol{r}-\boldsymbol{d}^{(2)}\right)\right. \\
& \left.-\psi_{m}^{(\overline{1} \overline{1} 1)}\left(\boldsymbol{r}-\boldsymbol{d}^{(3)}\right)-\psi_{m}^{(\overline{1} 1 \overline{1})}\left(\boldsymbol{r}-\boldsymbol{d}^{(4)}\right)\right\}, \\
\Psi_{3}(\boldsymbol{r})= & \frac{1}{2}\left\{\psi_{m}^{(111)}\left(\boldsymbol{r}-\boldsymbol{d}^{(1)}\right)-\psi_{m}^{(1 \overline{1} \overline{1})}\left(\boldsymbol{r}-\boldsymbol{d}^{(2)}\right)\right. \\
& \left.-\psi_{m}^{(\overline{1} \overline{1} 1)}\left(\boldsymbol{r}-\boldsymbol{d}^{(3)}\right)+\psi_{m}^{(\overline{1} 1 \overline{1})}\left(\boldsymbol{r}-\boldsymbol{d}^{(4)}\right)\right\},
\end{aligned}
$$


where $\psi_{m}^{v}(\boldsymbol{r})$ is the $d$-orbital function with the quantum number of $m$ and the polar axis $v$, and it can be chosen as a real function due to time-reversal symmetry. In this basis, the representations of twofold and threefold rotations, $R_{1 / 2}^{v}$ and $R_{n / 3}^{v}$, respectively, are given by

$$
\begin{gathered}
R_{1 / 2}^{(100)}=\left(\begin{array}{ccc}
-1 & 0 & 0 \\
0 & 1 & 0 \\
0 & 0 & -1
\end{array}\right) \\
R_{1 / 3}^{(111)}=e^{i(2 \pi / 3) m}\left(\begin{array}{ccc}
0 & 0 & 1 \\
1 & 0 & 0 \\
0 & 1 & 0
\end{array}\right)=\left\{R_{2 / 3}^{(111)}\right\}^{-1} .
\end{gathered}
$$

The representations for the other operations can be obtained as a matrix product of $R_{1 / 2}^{(100)}$ and $R_{1 / 3}^{(111)}$.

We discuss an effective Hamiltonian describing electronic states around the $\Gamma$ point by using the representations. Three functions $\Psi_{j}$ are degenerated at the $\Gamma$ point but they split into three at nonzero wave numbers. In the vicinity of the $\Gamma$ point, electronic states can be described by the $k$-linear Hamiltonian,

$$
H_{\text {eff }}=\hat{v}_{x} k_{x}+\hat{v}_{y} k_{y}+\hat{v}_{z} k_{z}
$$

where $\hat{v}_{\mu}$ is a $3 \times 3$ velocity matrix on the bases of $\Psi_{j}$ and we set the node to be the origin of energy. When we consider electronic states in the $k_{\mu}$ axis, the velocity matrix $\hat{v}_{\mu}$ represents the Hamiltonian, $H_{\mathrm{eff}}^{(1)}=\hat{v}_{\mu} k_{\mu}$, and thus it is invariant under $R_{1 / 2}^{\mu}$. Therefore, for instance, $\hat{v}_{x}$ can be represented by

$$
\begin{aligned}
\hat{v}_{x}= & v_{0 x}\left(\begin{array}{ccc}
1 & 0 & 0 \\
0 & 0 & 0 \\
0 & 0 & 1
\end{array}\right)+v_{1 x}\left(\begin{array}{ccc}
0 & 0 & 1 \\
0 & 0 & 0 \\
1 & 0 & 0
\end{array}\right) \\
& +v_{2 x}\left(\begin{array}{ccc}
0 & 0 & -i \\
0 & 0 & 0 \\
i & 0 & 0
\end{array}\right)+v_{3 x}\left(\begin{array}{ccc}
1 & 0 & 0 \\
0 & 0 & 0 \\
0 & 0 & -1
\end{array}\right) \\
& +v_{4 x}\left(\begin{array}{lll}
0 & 0 & 0 \\
0 & 1 & 0 \\
0 & 0 & 0
\end{array}\right) .
\end{aligned}
$$

In the pristine $\mathrm{CoSi}$, time-reversal symmetry is preserved besides crystal symmetries. Since all bases are chosen to be unchanged under time-reversal operation, the time-reversal operator for the Hamiltonian is $\mathcal{T} H(\boldsymbol{k}) \mathcal{T}^{-1}=H^{*}(-\boldsymbol{k})$. Thus time-reversal symmetry requires $v_{j x}$ to be nonzero only for $j=2$, i.e., $v_{j x}$ must be real. Moreover, the velocity operators in the other two directions are obtained by threefold rotation in Eq. (5). Therefore, the effective Hamiltonian is written by

$$
H_{\mathrm{eff}}=i v\left(\begin{array}{ccc}
0 & -k_{y} & k_{x} \\
k_{y} & 0 & -k_{z} \\
-k_{x} & k_{z} & 0
\end{array}\right)
$$

Here the Hamiltonian is obtained by setting $\theta=\pi / 2$ in the general $k$-linear Hamiltonian for threefold degenerate fermion [9] and equivalent to a model proposed in Ref. [38]. By using the data of the first-principles band, the velocity coefficient is estimated to be $v \sim 6.7 \times 10^{7} \mathrm{~cm} / \mathrm{s}$ around the $\Gamma$ point. The vector component of Bloch functions are given by

$$
\left|\psi_{0}\right\rangle=\frac{1}{k}\left(\begin{array}{c}
k_{z} \\
k_{x} \\
k_{y}
\end{array}\right), \quad\left|\psi_{ \pm}\right\rangle=\frac{1}{k \sqrt{2\left(k_{x}^{2}+k_{y}^{2}\right)}}\left(\begin{array}{c}
k_{x}^{2}+k_{y}^{2} \\
\pm i k k_{y}-k_{x} k_{z} \\
\mp i k k_{x}-k_{y} k_{z}
\end{array}\right),
$$

with energy $E=0$ and $\pm v k$, respectively. The velocity operators in the $x, y$, and $z$ directions map $\psi_{-}$onto $\psi_{0}$ and $\psi_{-}$as

$$
\begin{gathered}
\left\langle\psi_{0}\left|\left(\begin{array}{c}
\hat{v}_{x} \\
\hat{v}_{y} \\
\hat{v}_{x}
\end{array}\right)\right| \psi_{-}\right\rangle=\frac{v}{k \sqrt{2\left(k_{x}^{2}+k_{y}^{2}\right)}}\left(\begin{array}{c}
i k k_{y}+k_{x} k_{z} \\
i k k_{x}-k_{y} k_{z} \\
k_{x}^{2}+k_{y}^{2},
\end{array}\right) \\
\left\langle\psi_{-}\left|\left(\begin{array}{c}
\hat{v}_{x} \\
\hat{v}_{y} \\
\hat{v}_{z}
\end{array}\right)\right| \psi_{-}\right\rangle=\frac{v}{k}\left(\begin{array}{c}
k_{x} \\
-k_{y} \\
-k_{z}
\end{array}\right) .
\end{gathered}
$$

Any velocity operator has zero as matrix elements between $\psi_{-}$and $\psi_{+}$. Therefore, the transition between the lower band and the upper band is prohibited, and thus it has no contribution to the dynamical conductivity and leads to the characteristic structure in Figs. 3(a) and 4(b).

We analyze the prohibition from the point of view of symmetry by using the low-energy model for multifold chiral fermion in Eq. (8). The effective Hamiltonian has skew symmetry, which is an antisymmetry and represented by $\left\{H_{\text {eff }}(\boldsymbol{k})\right\}^{*}=-H_{\text {eff }}(\boldsymbol{k})$. Thus, electronic states in the upper and lower bands are transformed to each other $\left|\psi_{+}(\boldsymbol{k})\right\rangle=$ $\left|\psi_{-}(\boldsymbol{k})\right\rangle^{*}$, and those in the middle and flatband is invariant $\left|\psi_{0}(\boldsymbol{k})\right\rangle=\left|\psi_{0}(\boldsymbol{k})\right\rangle^{*}$. The skew symmetry leads to the absence of velocity matrix component between the upper and lower bands $\quad\left\langle\psi_{+}(\boldsymbol{k})\left|\hat{v}_{\mu}\right| \psi_{-}(\boldsymbol{k})\right\rangle=-\left\{\left\langle\psi_{-}(\boldsymbol{k})\left|\hat{v}_{\mu}\right| \psi_{+}(\boldsymbol{k})\right\rangle\right\}^{*}=0$ because the velocity is a Hermite operator. In the realistic material, this symmetry is not preserved globally because of the nonlinear term with the wave vector in the Hamiltonian. Therefore, the electronic transition between the upper and lower bands increases with increasing in $|\boldsymbol{k}|$.

The prohibition of electronic transition is a characteristic feature of the threefold chiral fermion in comparison with Weyl and Dirac semimetals, the other systems with the linear dispersion. The skew symmetry leads to the correlation between electronic states in the upper and lower cones and restricts the electronic transition between them. In Dirac and Weyl semimetals, on the other hand, the intercone transition is relevant in the low-frequency electronic excitation. The Dirac and Weyl Hamiltonian breaks the skew symmetry and provides no restriction to the excitation process.

\section{CONCLUSION}

We have investigated the dynamical conductivity of CoSi, a topological semimetal holding multifold chiral fermions, by using first-principles band calculation and linear response theory. The spectrum of dynamical conductivity $\sigma_{1}(\omega)$ exhibits the dip and peak structures at some photon frequency $\omega$. We investigate the relation between these structures and the electronic structure. The peak structures in $1 \mathrm{eV}<\hbar \omega$ are corresponding to the transition between the local maximum and minimum of the occupied and unoccupied bands, respectively. The peak structure at $\hbar \omega=650 \mathrm{meV}$, on the 
other hand, is attributed to the electronic transition between parallel bands and shows the flat-top structure due to the spin-split.

In the lower photon energy region, the spectrum reflects the property of multifold chiral fermions. The dynamical conductivity linearly increases with the photon energy and turns down at $\hbar \omega=170 \mathrm{meV}$. This behavior is attributed to not only the band structure but also the chirality of multifold fermion at the $\Gamma$ point. We show the prohibition of transition between the upper and lower bands of threefold chiral fermion and that the prohibition leads to the decrease in the dynamical conductivity. Then the partially filled linear dispersion at the $\mathrm{R}$ point contributes to the increase again at $\hbar \omega=200 \mathrm{meV}$. The two multifold chiral fermionic states at the $\Gamma$ and $\mathrm{R}$ points coproduce a dip structure in the spectrum of dynamical conductivity of CoSi.

\section{ACKNOWLEDGMENTS}

The author would like to thank Y. Asano for helpful discussions. This work was supported by the JSPS KAKENHI (Grant No. JP15H05852).
[1] S. Murakami, New J. Phys. 9, 356 (2007).

[2] X. Wan, A. M. Turner, A. Vishwanath, and S. Y. Savrasov, Phys. Rev. B 83, 205101 (2011).

[3] K.-Y. Yang, Y.-M. Lu, and Y. Ran, Phys. Rev. B 84, 075129 (2011)

[4] A. A. Burkov and L. Balents, Phys. Rev. Lett. 107, 127205 (2011).

[5] M. Neupane, S. Xu, R. Sankar, N. Alidoust, G. Bian, C. Liu, I. Belopolski, T.-R. Chang, H.-T. Jeng, H. Lin, A. Bansil, F. Chou, and M. Z. Hasan, Nat. Commun. 5, 3786 (2014).

[6] S. Borisenko, Q. Gibson, D. Evtushinsky, V. Zabolotnyy, B. Büchner, and R. J. Cava, Phys. Rev. Lett. 113, 027603 (2014).

[7] B. J. Wieder, Y. Kim, A. M. Rappe, and C. L. Kane, Phys. Rev. Lett. 116, 186402 (2016).

[8] B. Bradlyn, J. Cano, Z. Wang, M. G. Vergniory, C. Felser, R. J. Cava, and B. A. Bernevig, Science 353, aaf5037 (2016), https: //science.sciencemag.org/content/353/6299/aaf5037.full.pdf.

[9] B. Bradlyn, L. Elcoro, J. Cano, M. G. Vergniory, Z. Wang, C. Felser, M. I. Aroyo, and B. A. Bernevig, Nature 547, 298 (2017).

[10] P. Tang, Q. Zhou, and S.-C. Zhang, Phys. Rev. Lett. 119, 206402 (2017).

[11] B. Bradlyn, L. Elcoro, M. G. Vergniory, J. Cano, Z. Wang, C. Felser, M. I. Aroyo, and B. A. Bernevig, Phys. Rev. B 97, 035138 (2018).

[12] G. Chang, B. J. Wieder, F. Schindler, D. S. Sanchez, I. Belopolski, S.-M. Huang, B. Singh, D. Wu, T.-R. Chang, T. Neupert et al., Nat. Mater. 17, 978 (2018).

[13] G. Chang, S.-Y. Xu, B. J. Wieder, D. S. Sanchez, S.-M. Huang, I. Belopolski, T.-R. Chang, S. Zhang, A. Bansil, H. Lin, and M. Z. Hasan, Phys. Rev. Lett. 119, 206401 (2017).

[14] M.-Á. Sánchez-Martínez, F. de Juan, and A. G. Grushin, Phys. Rev. B 99, 155145 (2019).

[15] N. B. Schröter, D. Pei, M. G. Vergniory, Y. Sun, K. Manna, F. de Juan, J. A. Krieger, V. Süss, M. Schmidt, P. Dudin et al., Nat. Phys., 1 (2019).

[16] F. Flicker, F. de Juan, B. Bradlyn, T. Morimoto, M. G. Vergniory, and A. G. Grushin, Phys. Rev. B 98, 155145 (2018).

[17] D. Rees, K. Manna, B. Lu, T. Morimoto, H. Borrmann, C. Felser, J. Moore, D. H. Torchinsky, and J. Orenstein, arXiv:1902.03230.

[18] P. Dutta and S. K. Pandey, Comput. Cond. Matter 16, e00325 (2018).
[19] D. A. Pshenay-Severin, Y. V. Ivanov, and A. T. Burkov, J. Phys.: Condens. Matter 30, 475501 (2018).

[20] D. Takane, Z. Wang, S. Souma, K. Nakayama, T. Nakamura, H. Oinuma, Y. Nakata, H. Iwasawa, C. Cacho, T. Kim, K. Horiba, H. Kumigashira, T. Takahashi, Y. Ando, and T. Sato, Phys. Rev. Lett. 122, 076402 (2019).

[21] Z.-C. Rao, H. Li, T.-T. Zhang, S.-J. Tian, C.-H. Li, B.-B. Fu, C.Y. Tang, L. Wang, Z.-L. Li, W.-H. Fan et al., Nature (London) 567, 496 (2019).

[22] T. Habe and M. Koshino, Phys. Rev. B 98, 125201 (2018).

[23] M. B. Schilling, L. M. Schoop, B. V. Lotsch, M. Dressel, and A. V. Pronin, Phys. Rev. Lett. 119, 187401 (2017).

[24] P. Giannozzi, S. Baroni, N. Bonini, M. Calandra, R. Car, C. Cavazzoni, D. Ceresoli, G. L. Chiarotti, M. Cococcioni, I. Dabo, A. Dal Corso, S. de Gironcoli, S. Fabris, G. Fratesi, R. Gebauer, U. Gerstmann, C. Gougoussis, A. Kokalj, M. Lazzeri, L. Martin-Samos et al., J. Phys.: Condens. Matter 21, 395502 (2009).

[25] F. Ishii, H. Kotaka, and T. Onishi, Spin-orbit interaction effects in the electronic structure of b20-type cosi: Firstprinciples density functional study, in Proceedings of the International Conference on Strongly Correlated Electron Systems (SCES2013) (2014), https://journals.jps.jp/doi/pdf/10. 7566/JPSCP.3.016019.

[26] T. Ando, Y. Zheng, and H. Suzuura, J. Phys. Soc. Jpn. 71, 1318 (2002).

[27] V. P. Gusynin and S. G. Sharapov, Phys. Rev. B 73, 245411 (2006).

[28] M. Koshino and T. Ando, Phys. Rev. B 77, 115313 (2008).

[29] C. J. Tabert and J. P. Carbotte, Phys. Rev. B 93, 085442 (2016).

[30] S. P. Mukherjee and J. P. Carbotte, Phys. Rev. B 95, 214203 (2017).

[31] S. Ahn, E. J. Mele, and H. Min, Phys. Rev. Lett. 119, 147402 (2017).

[32] S. Barati and S. H. Abedinpour, Phys. Rev. B 96, 155150 (2017).

[33] J. Ebad-Allah, J. F. Afonso, M. Krottenmüller, J. Hu, Y. Zhu, Z. Mao, J. Kuneš, and C. Kuntscher, Phys. Rev. B 99, 125154 (2019). 
[34] T. Stauber, N. M. R. Peres, and A. K. Geim, Phys. Rev. B 78, 085432 (2008).

[35] K. F. Mak, M. Y. Sfeir, Y. Wu, C. H. Lui, J. A. Misewich, and T. F. Heinz, Phys. Rev. Lett. 101, 196405 (2008).
[36] A. A. Mostofi, J. R. Yates, Y.-S. Lee, I. Souza, D. Vanderbilt, and N. Marzari, Comput. Phys. Commun. 178, 685 (2008).

[37] S. P. Mukherjee and J. P. Carbotte, J. Phys.: Condens. Matter 29, 425301 (2017).

[38] J. L. Mañes, Phys. Rev. B 85, 155118 (2012). 